\title{
Modelica-based modeling and simulation of satellite On-orbit deployment and attitude control
}

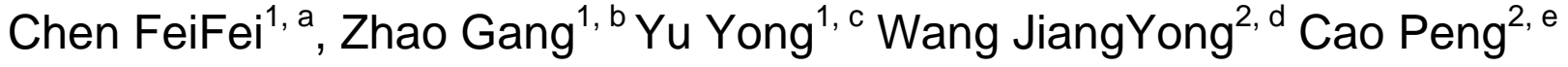 \\ ${ }^{1}$ School of Mechanical Engineering and Automation, Beijing University of Aeronautics and \\ Astronautics, Beijing 100191, China; \\ ${ }^{2}$ Institute of Telecommunication Satellite, China Academy of Space Technology, Beijing 100194, \\ China. \\ a Chenfly@buaa.edu.cn, bzhaog@buaa.edu.cn, ${ }^{\mathrm{b}}$ yuyong@buaa.edu.cn, \\ d caopeng@spacechina.com , e wangjy501@cast.cn
}

Keywords: modelica; multi-domain modeling and simulation; on-orbit deployment; attitude control

\begin{abstract}
In order to reflect the dynamic characteristics of satellite on-orbit deployment more precisely, a Modelica-based modeling and simulation of satellite on-orbit deployment and attitude control was achieved. Form Modelica standard library, world component was extended, this simulation system integrates Space perturbation factors and Celestial time scales. Based on Modelica Object-oriented character, synchronous deployment model of satellite solar panel was developed, satellite on-orbit deployment by integrating Space perturbation functions and keplerian orbit parameters were achieved. Based on Modelica united modeling character, between direct and inverse model, the inverse dynamic attitude control system was constructed. The simulation of satellite On-orbit deployment and earth-oriented attitude control, verifying whether the satellite on-orbit deployment demands the overall design of satellite, supporting rapid prototyping during all phases (project proposal, subsystem, system, etc.).
\end{abstract}

\section{Introduction}

Satellite On-orbit deployment and attitude control is an organic composition consists of the area of different disciplines such as machine, electricity, and control. The area of different disciplines reflects the multi-domain system, high precision and high confidence level simulation, which requires the satellite model reflects its multi-domain characteristics. However, Most of modeling and simulation with multi-body, such as solar wings, uses ADMAS, DAMS etc. These soft-wares modeling system mainly to single mechanical field, taking no account of space environment perturbation, electro-mechanical coupling factors and so on. The article based on Modelica (develop environment is Mworks) modeling and simulation for satellite deployment on-orbit and attitude control is achieved. The model contains mechanical, electrical, control, magnetic field.

\section{The overall framework of the simulation model}

The system of satellite on-orbit deployment and attitude control (Fig. 1), consists of four parts:

1) Extended World model. Determining the starting time of the simulation, selecting the orbit environment of satellite deployment on-orbit. The user can define the initial Keplerain parameters. Keplerian_orbit_para component provides the initial position simulation of direct/inverse satellite model.

2) Environment perturbation component. Inheriting perturbation function determined by extended world model, acting on both direct and inverse model. Magnetos model can also inherit from extended world model, obtaining magnetic field of position of satellite.

3) Inverse system part. The mission manager component output the desired angular velocity and desired attitude quaternion, acting on the inverse satellite model with actuator. The inverse of satellite with actuator output moment of reaction, preliminarily adjusting the attitude of satellite. 
4) Direct control part. The Aero-spacecraft orbit control system (AOCS) receives the actual attitude from the sensor model, desired angular velocity and desired attitude quaternion. At the same time, AOCS produces control electric current and magnetic field, achieving more precisely control for satellite attitude.

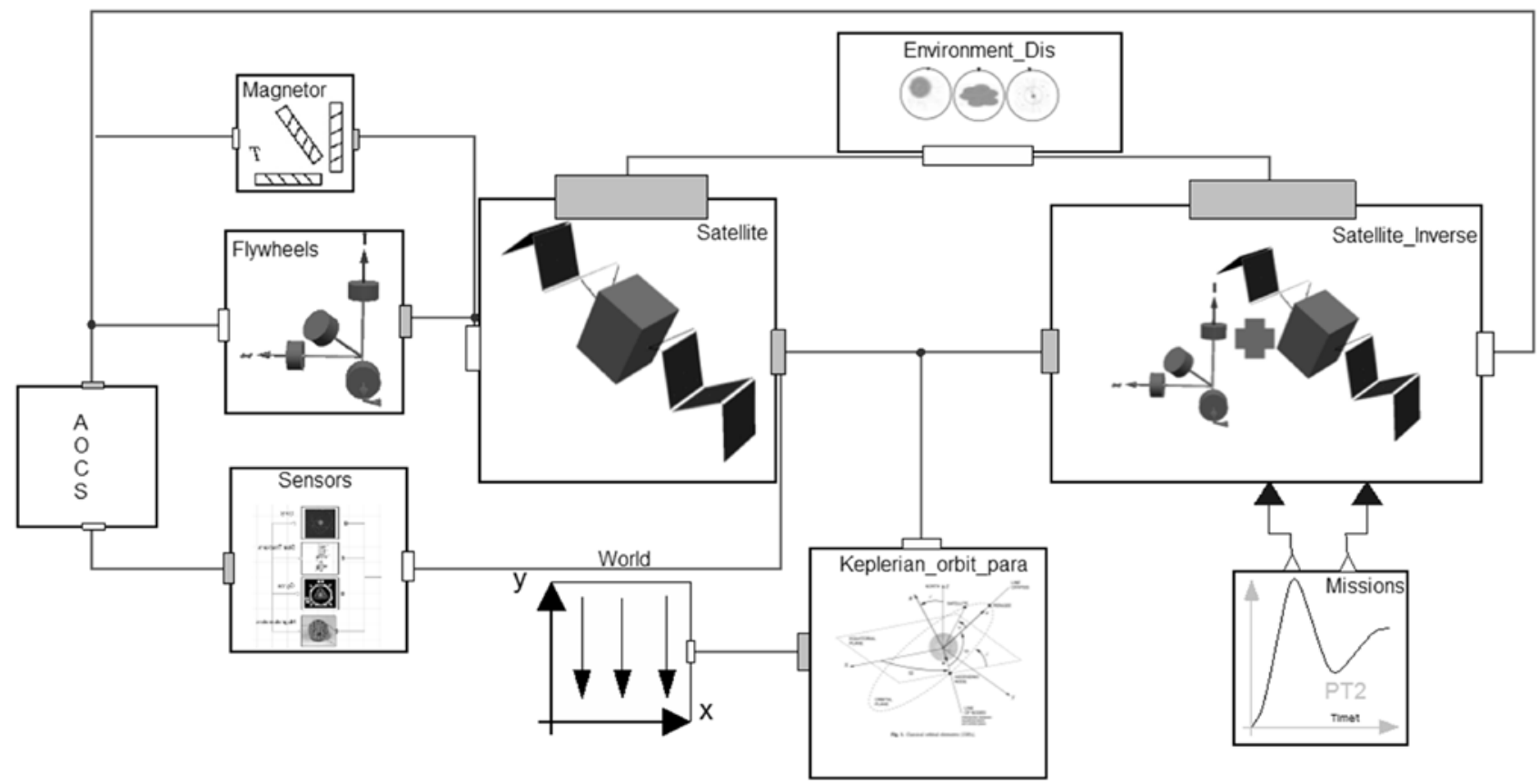

Fig. 1 Satellite On-orbit deployment and attitude control simulation system

\section{The process of modeling}

\subsection{Extended World Component.}

World component is one of the most important components in Modelica mechanical library. The World Coordinate System is modeled from World Component, however, it did not provide details for the Earth's gravity field, for the Earth's Magnetic field, for the atmospheric density model and so on, which need to be extended. As can be seen from the Fig. 2 [2].

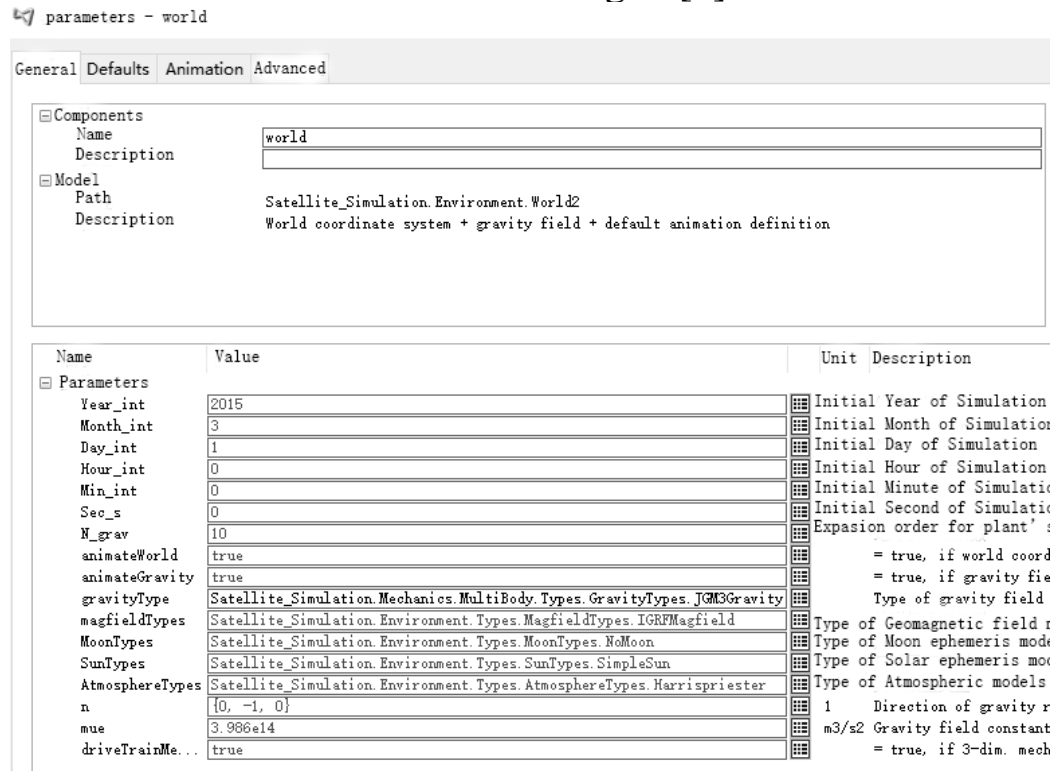

Fig. 2 User interface for the Extended world model

As is well known, the earth's gravitational potential ${ }^{U_{g}}$ may be described by the function.

$U_{g}(r, \theta, \lambda)=-\frac{\mu}{r}\left\{1+\sum_{n=2}^{\infty}\left(\frac{R_{e}}{r}\right)^{n} J_{n} P_{n}(\cos (\theta))+\sum_{n=2 m=1}^{\infty} \sum_{n}^{n}\left(\frac{R_{e}}{r}\right)^{n} P_{n}^{m}(\cos (\theta))\left(C_{n}^{m} \cos (m \lambda)+S_{n}^{m} \sin (m \lambda)\right)\right\}$ 
Where ${ }^{P_{n}^{m}}$ are the Legendre Polynomials. ${ }^{R_{e}}$ Is the mean equatorial Earth radius, $r, \theta$ and $\lambda$ are the point's spherical coordinates and coefficients $J_{n}, C_{n}^{m}, S_{n}^{m}$ are the zonal, sectoral and tesserae coefficients. Depending on the mission characteristics and on the purpose of attitude control simulations, a satisfactory approximation can be obtained by choosing the order of the expansion in a suitable way. The Earth gravitational field components (expressed in spherical coordinates) are then given by.

$$
g=-\nabla U_{g}=-\left\{\frac{\partial U_{g}}{\partial r}, \frac{1}{r} \frac{\partial U_{g}}{\partial \theta}, \frac{1}{r \sin (\theta)} \frac{\partial U_{g}}{\partial \lambda}\right\}
$$

Similar models for the Earth's Magnetic field, the atmospheric density and the Sun and Moon position have been implemented in Modelica according to [3].

\subsection{Modeling and implementation of satellite deployment on-oribit}

\subsubsection{The solar wings synchronization model}

The solar wings synchronization model consists of sub-models: the solar wing multi-body model, driving element model, Locking mechanism model, Synchronization mechanism model, Clearance Joint model. With these sub-models, the solar wing synchronization model is modeled in an object-oriented way (Fig. 4).

Modelica Standard Library (MSL) is used to model the solar wings multi-body model. Satellite base, connect link, solar plane is simplified as rigid model. Complex mechanism, such as Clearance Joint, Synchronization mechanism were modeled according to the structural characteristics.

The driving element for solar wings spreading is Spiral spring in joint, the size of the deformation angle is proportional to the torque, and the running torque is related to wide of Spiral spring, the thickness of Spiral spring, the length of Spiral spring, the bending strength of Spiral spring. The properties of Spiral spring can be simplified as the rigidity coefficient of spiral spring $K$, damping coefficient of spiral spring ${ }^{C}$, preloaded angle of spiral spring ${ }^{\phi_{0}}$, spread angle of spiral spring ${ }^{\phi_{w}}$. The equation of torque expression is (3).

$$
T=K\left(\phi_{0}-\phi_{w}\right)-C \dot{\phi}
$$

The commonly applied locking mechanism modeling method is used to model locking mechanism [4] [5]. Defining step function STEP to detect the behavior of locked detecting. When the value of STEP is one, starting lock. Defining double side collision function BISTOP, when the value of STEP is 1 , BISTOP taking effect. Their expressions are (4).

$$
\begin{aligned}
& \operatorname{STEP}\left(\theta, x_{1}, l, x_{2}, h\right)=\left\{\begin{array}{c}
l-(l-h)\left(\frac{\theta-x_{1}}{x_{2}-x_{1}}\right)^{2}\left(3-2 \frac{\theta-x_{1}}{x_{2}-x_{1}}\right) \\
x_{1} \leq \theta \leq x_{2} \\
\theta>x_{2}
\end{array}\right. \\
& \operatorname{BISTOP}\left(\theta, \dot{\theta}, x_{3}, x_{4}, k, e, c, d\right)=\left\{\begin{array}{cc}
{\left[k\left(x_{3}-\theta\right)^{e}-\dot{\theta} \operatorname{STEP}\left(\theta, x_{3}-d, c, x_{3}, 0\right), 0\right]_{\max }} & \theta<x_{3} \\
0 & x_{3}<\theta<x_{4} \\
{\left[-k\left(\theta-x_{4}\right)^{e}-\dot{\theta} \operatorname{STEP}\left(\theta, x_{4}, 0, x_{4}+d, c\right), 0\right]_{\min }} & \theta>x_{4}
\end{array}\right.
\end{aligned}
$$

Where $\theta$ is spread angle of part, $\dot{\theta}$ is spread angular velocity of part, ${ }^{x_{1}, x_{2}}$ respectively are the low threshold and the high threshold of Angular displacement variables in STEP function; ${ }_{3}^{x_{3}, x_{4}}$ respectively are the low threshold and the high threshold of Angular displacement variables in double side collision function BISTOP, $k$ is rigidity coefficient, $e$ is the rigidity index, ${ }^{c}$ is damping coefficient, $d$ is damping incremental distance.

Closed configuration loop (CCL) is used as synchronous deployment control mechanism of solar wings, consists of engaged wheel and rope, which working condition as Fig. 3. 


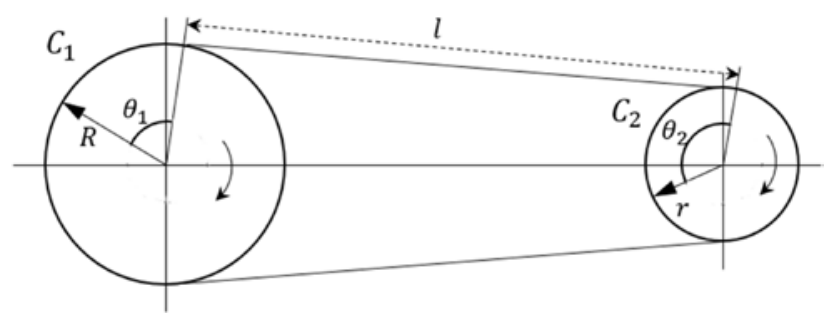

Fig. 3 Synchronization mechanism

$$
\Delta l=C_{1}-C_{2}=R \theta_{1}-r \theta_{2}
$$

Where $C_{1}$ is the arc length of the engaged wheel, the radius is $R$, which rotate of angle $\theta_{1}, C_{2}$ is the arc length of the engaged wheel, the radius is $r$, which rotate of angle $\theta_{2}, \Delta l$ is the deformation of rope, stretch is positive, shorten is negative.

$$
\begin{gathered}
F_{1}=\left\{\begin{array}{cc}
K_{l} \Delta l+F_{0} & \Delta l>-\frac{F_{0}}{K_{l}} \\
0 & \Delta l \leq-\frac{F_{0}}{K_{l}}
\end{array}\right. \\
F_{2}=\left\{\begin{array}{cc}
F_{0}-K_{l} \Delta l & \Delta l<\frac{F_{0}}{K_{l}} \\
0 & \Delta l \geq \frac{F_{0}}{K_{l}}
\end{array}\right.
\end{gathered}
$$

Clearance joint is modeled to make the model more reliable [6]. The method of collision joint is applied, which considers the shaft and bearing as two collision part. The dynamic characteristic depends on the collision force between clearances. Modelica language is used to describe the behavior of clearance joint. Clearance vector model is used to describe collision contact force model between clearance parts.

Collision contact force model, which apply the improved method of nonlinear continuous contact collision hybrid force model. Friction model, which applies modified coulomb friction model. The expression is respectively (7).

$$
\begin{array}{r}
F_{n}=\left\{\begin{array}{cc}
K \delta^{n}+D \dot{\delta} & \delta \geq 0 \\
0 & \delta<0
\end{array}\right. \\
F_{t}=-\mu\left(v_{t}\right) F_{n} \frac{v_{t}}{\left|v_{t}\right|}
\end{array}
$$

Where $F_{n}$ is normal contact force, $F_{t}$ is tangent friction force, $D$ is the damping coefficient during collision. $\dot{\delta}$ is opposite speed, $K$ is contact-restored coefficient, $\delta$ is penetration depth, ${ }_{t}{ }$ is Tangential speed, $u\left(v_{t}\right)$ is dynamic friction coefficient.

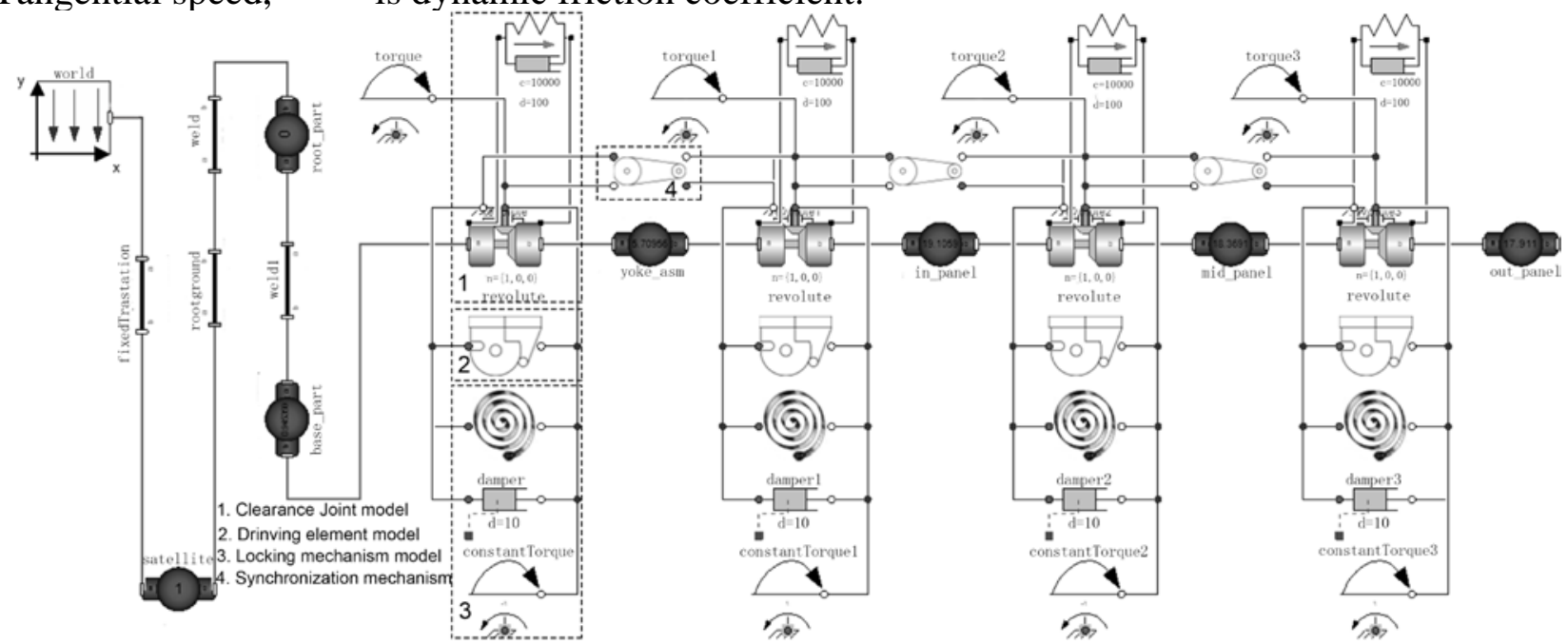

Fig. 4 Synchronous deployment model of unilateral solar panel 


\subsubsection{The implementation of solar wings deployment on-orbit}

First, defining the movement of the satellite relative to the ECI coordinate system through Keplerian_orbit_para component. Then, corresponding space environment perturbation factors is superimposed. Last, Freemotion component is used to achieve the orbit dynamics of satellite.

1) Defining the initial time of satellite deployment on-orbit

Satellite state representation in terms of classical orbital elements (Keplerian parameters) will be denoted as $x_{C O E}=[a, e, i, \Omega, \omega,(M-n t)]^{T}$.Where,a:semi-major axis,[m]; e :eccentricity,[dimensionless]; i: inclination[rad]; $\Omega$ : right ascension of the ascending node[rad]; ${ }^{\omega}:$ argument of perigee[rad]; ${ }^{n}$ : mean motion, [rad/s].

The conversion relations between Kepler's parameter and position speed under ECI as shown (8).

$$
\begin{gathered}
x_{E C I}=\left[\begin{array}{ll}
r^{T} & v^{T}
\end{array}\right]^{T} \\
{\left[\begin{array}{c}
x_{\text {orb }} \\
y_{\text {orb }}
\end{array}\right]=\left[\begin{array}{c}
a \cos (E)-a E \\
a \sin (E) \sqrt{1-e^{2}}
\end{array}\right]} \\
{\left[\begin{array}{c}
v_{x, \text { orb }} \\
v_{y, \text { orb }}
\end{array}\right]=\left[\begin{array}{c}
-\frac{a^{2} n}{|r|} \sin (E) \\
\frac{a^{2} n}{|r|} \sqrt{1-e^{2}} \cos (E)
\end{array}\right]} \\
{\left[\begin{array}{c}
x_{\text {orb }} \\
y_{\text {orb }} \\
0
\end{array}\right]=R_{z X z}\left(x_{C O E}\right)\left[\begin{array}{l}
x \\
y \\
z
\end{array}\right]}
\end{gathered}
$$

$|r|=\sqrt{x_{o r b}{ }^{2}+y_{o r b}{ }^{2}}=\sqrt{r^{T} r}, R_{z x z}\left(x_{C O E}\right)=R_{Z}(w) R_{X}(i) R_{Z}(\Omega)$ is Gaussian vector for conversion between equatorial planet coordinate and orbital plane coordinate.

To improve accuracy in the computation of Keplerian orbital motion, the Keplerian orbit parameter used as state variables integrated in body component [7].

The specific implementation process as follows:

a) Adding Keplerian orbital parameter ${ }^{a, e, i, \Omega, \omega, M}$ as new model variables.

b) Adding the equations relating Keplerian orbital parameter to ECI coordinates.

c) Switching the stateselect attribute for the $r$ and v vectors of the Body model to StateSelect.avoid, and for the Keplerian orbital parameter to StateSelect.prefer.

Modelica compiler tool priority to choose Keplerian orbital parameter as state variable for data interaction. Comparing to position and velocity vector, Keplerian orbital parameter has low order of magnitudes, so the simulation accuracy would be improved. The data interaction process is shown as Fig. 5.

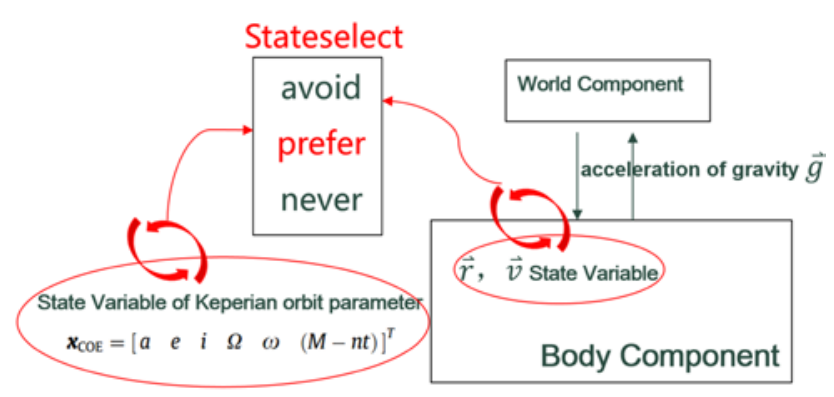

Fig. 5 The sketch map of State variables interaction

2) Superposition of space perturbation

Perturbation functions in world component is inherited, Environment perturbation component acts on each body after packaging (Fig. 6). The benefit of this way is selectivity open/close corresponding perturbation factor of component, to improve scalability of the model [2]. 


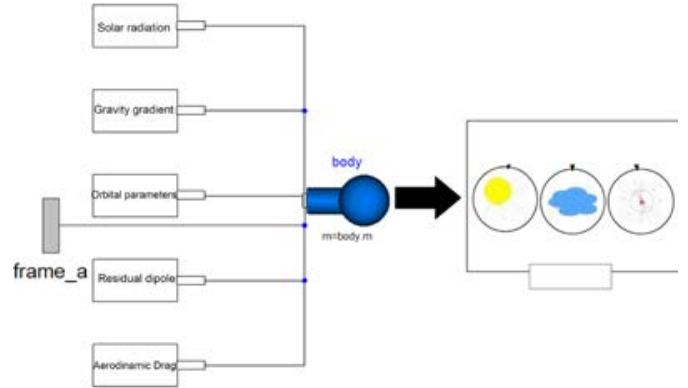

Fig. 6 Layout of body model with Space perturbation factors unilateral solar panel

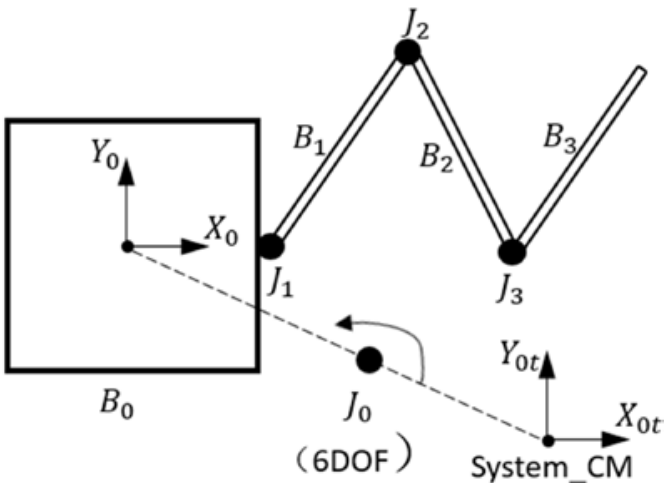

Fig. 7 On-orbit deployment of

3) The implement of orbit dynamics of satellite

The details of orbit dynamics of satellite as follows, Fig. 7. The base is the first body of the space robotic system (called ${ }^{B_{0}}$ ), from which the solar wings $\left({ }_{1}, B_{2}, B_{3}\right)$ are serially connected with. The link among Solar wings is represented as $J_{1}, J_{2}, J_{3}$. The relative movement between base and ECI is represented by ${ }^{J_{0}}$. The base movement on-orbit is the foundation of the solar wings deployment on-orbit. A 6 DOF joint $\left({ }^{J_{0}}\right)$ is used to denote the constraint between base and ECI. A free motion joint FreeMotion (the path is Modelica standard library is MultiBody.Joints.FreeMotion) [8]

\subsection{Modeling and implement of satellite attitude control}

Satellite attitude control is achieved by Sensors, Actuators and controller together. Flywheel components and magnetic torque actuator are used as actuators. Motor, Driver, bearing friction, transmission joint and wheel body is consisted of Flywheel components Modelica model. As Fig. 8.

The modeling process of Magnetic torque actuator is according to [9].

The sensor Model, such as, the rate gyroscope, earth sensor, sun sensor and start sensor, is modeled according the present document of sensor modeling method [10]. The Modelica language is used to achieve these. The standard Modelica sensor is used to get value of sensor for the simplified sensor model. Due to the Modelica united modeling character, between direct and inverse model, the inverse dynamic attitude control system is constructed [11] (Fig. 9).

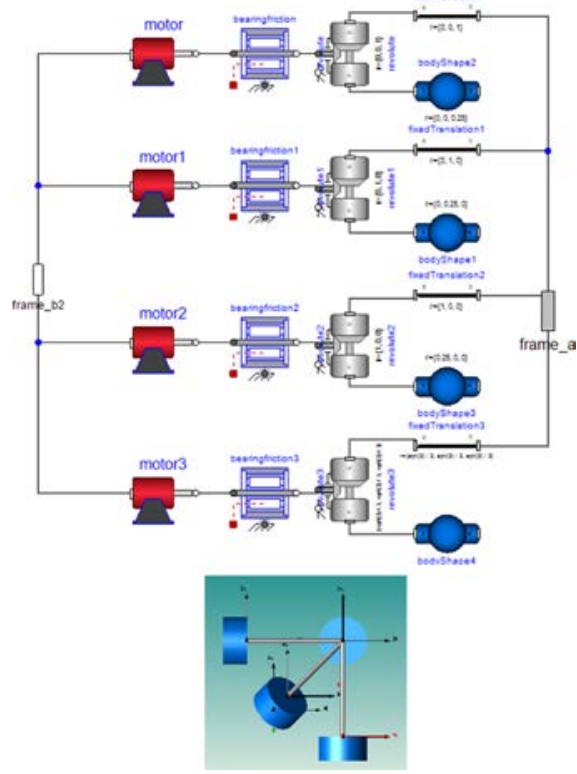

Fig. 8 Multi-domain model of flywheels and 3D display

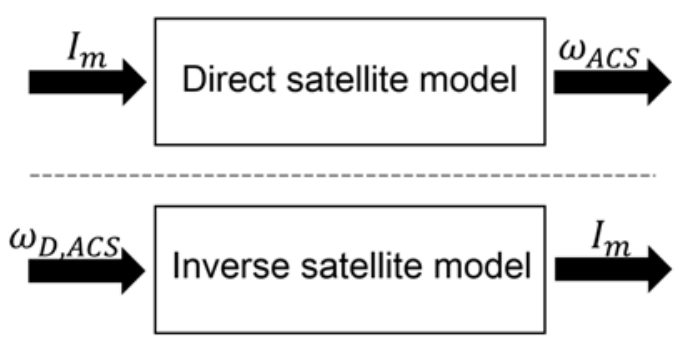

Fig. 9 Top level view of direct and inverse satellite model

The motor currents are used to drive the reaction wheels of the satellite, from which in turn the rotation of the satellite is determined by the solution of a differential-algebraic equation, which is the standard task of satellite simulators. However, for advanced control systems, the inverse of this model 
is needed, as shown in the lower half of Fig. 9. The previously given motor currents $I_{m}$ should now be computed from the desired angular velocity ${ }^{\omega_{D, A C S}}$.The inverse models may be automatically generated from the direct models in Modelica.

\section{Analysis of simulation result}

\subsection{Simulation of satellite deploying on-orbit}

The initial parameters of simulation is shown as table. I.

Table 1 Initial key simulation parameters

\begin{tabular}{|c|c|}
\hline \multicolumn{1}{|c|}{ Modeling object } & \multicolumn{1}{c|}{ Key simulation parameters } \\
\hline Satellite and solar wings multi-body model & Mass and moment of inertia were imported \\
\hline Synchronizaion mechanism model & \multicolumn{1}{c|}{ Flexibility coefficient $0.015 \mathrm{~mm} / \mathrm{N}$} \\
\hline \multicolumn{1}{|c|}{ Driving element model } & $\begin{array}{l}\text { Spiral spring is used, stiffness of joint } \\
\text { 0.0066Nm/deg }\end{array}$ \\
\hline Locking mechanism model & $\begin{array}{l}\text { Except angle of root joint } 90 \mathrm{deg}, \text { change } \\
\text { angle of locking } 0.1 \mathrm{deg}\end{array}$ \\
\hline Clearance joint model & $\begin{array}{l}\text { contact-restored coefficient,1e5Nm/deg, } \\
\text { damping coefficient } 100 \mathrm{Nms} / \mathrm{deg}, \quad \text { joint } \\
\text { clearance } 0.1 \mathrm{~mm}\end{array}$ \\
\hline Initial rotate angle of joints & $\begin{array}{l}\text { Initial rotate angle of root joint } 461 \mathrm{deg}, \text { initial } \\
\text { rotate angle among joints } 515 \mathrm{deg}\end{array}$ \\
\hline
\end{tabular}

The relating results are as follows:

a) Satellite deploying on-orbit has little effects on attitude of satellite, as Fig. 11 shows.

b) Fig. 12 shows population proportion of the spread angle of joint 1 between the spread angle of join 2,3,4 is 1:2 under the coordinate effect of Synchronization mechanism. At time $10.9 \mathrm{~s}$, locking behavior of model was happened, appearing a small shock, At the end, time 12.2s, model tended to become stable.

c) Fig. 13, shows the angular acceleration of link and solar plane. Because of the collision of Spiral spring, the angular.
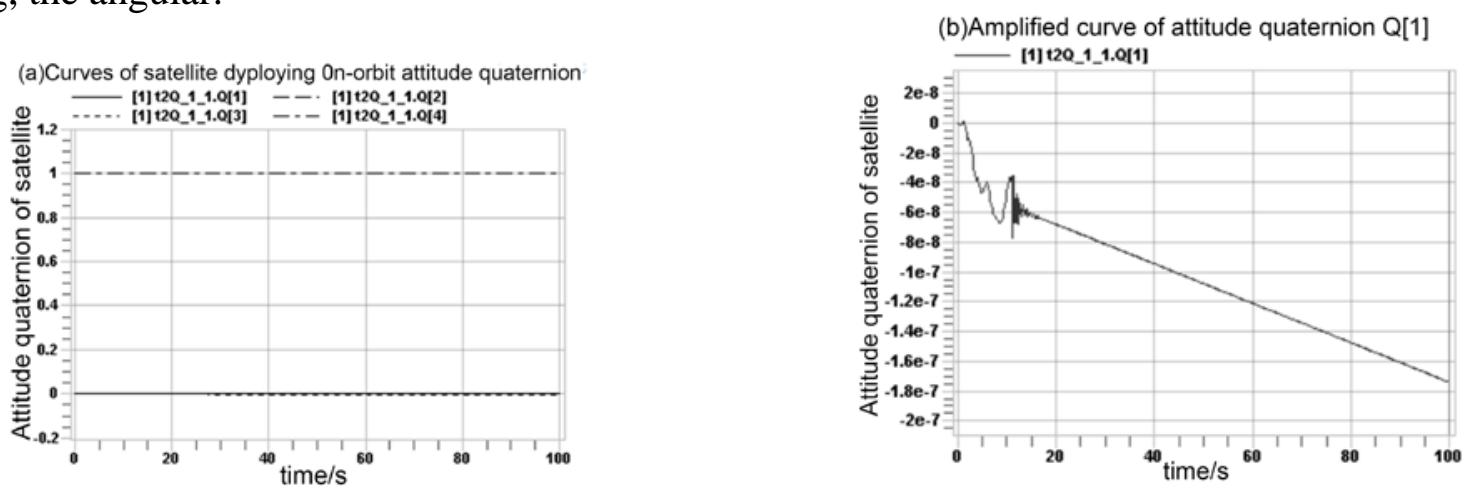

Fig.11 Curves of satellite on-orbit deployment attitude quaternion and amplified curve of attitude quaternion Q[1] 


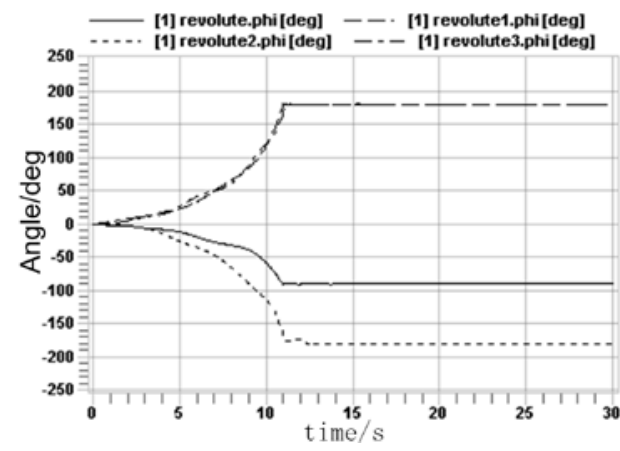

Fig.12 Curves of connect link and solar plane spread Angle Fig.13 Curve of connect link angular acceleration

\subsection{Simulation of satellite attitude control}

The earth-oriented attitude control is simulated, when the satellite is on-orbit. Simulation date is on March 1, 2015 UTC. Initial Keplerian parameters are: Semi-major axis ${ }^{a}: 421666 \mathrm{~km}$. Eccentricity ${ }^{e}$ : 0.0004 . Inclination ${ }^{i}: 0.02^{\circ}$. RA ascend $\Omega 0.0^{\circ}$. Arg. of perigee $\omega 0.0^{\circ}$. Mean anomaly $M 0.0^{\circ}$. The Earth's gravity field is JGM-3 model. The solar radiation, atmospheric damping and so on are considered.

Fig. 14 shows the change of attitude quaternion of satellite Earth-oriented in a day and corresponding trajectory and attitude of the satellite at 32832s.
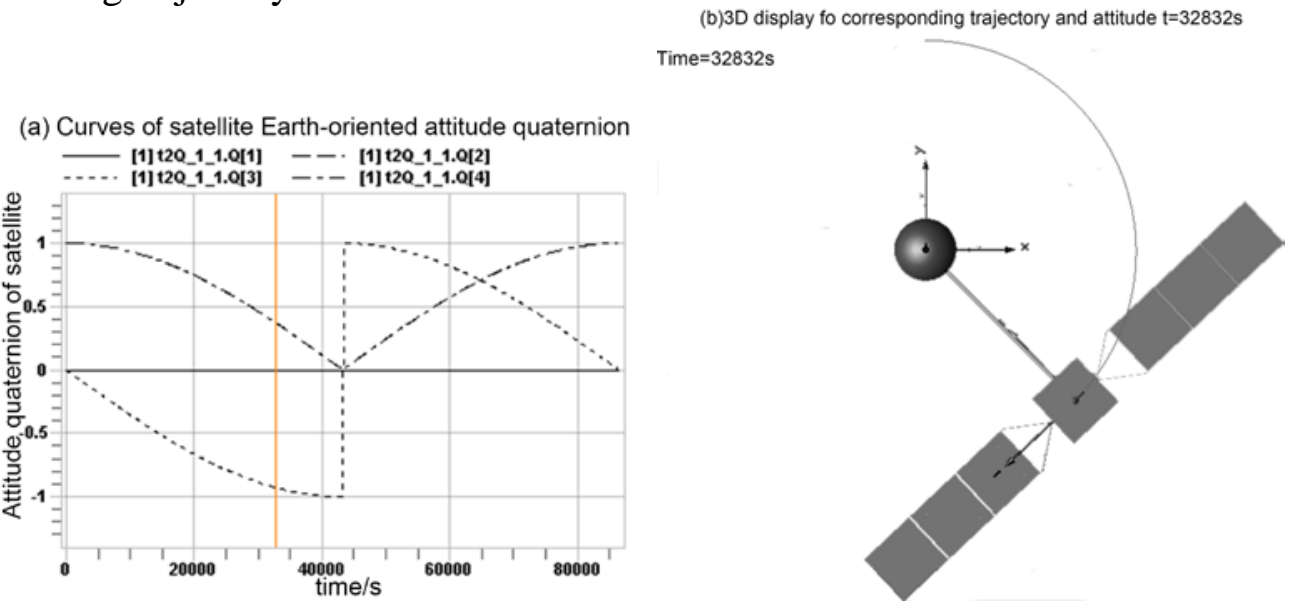

Fig. 14 Curves of satellite Earth-oriented attitude quaternion and 3D display of corresponding trajectory and attitude $(\mathrm{t}=32832 \mathrm{~s})$

\section{Summary}

A Modelica-based modeling and simulation of satellite on-orbit deployment and attitude control is achieved, the sub-models in system is reusable. The result of simulation shows:

a) Both Space environment perturbation and electromechanical coupling factor were considered, the influences on satellite deployment on-orbit are shown.

b) The spread time and angle of solar wings are predicted. Besides, the correspondence between spread angle and time and the ending velocity and acceleration was simulated, which could be used for the overall design of aero-spacecraft.

c) The inverse dynamic attitude control system was constructed, which could be used for the simulation of earth-oriented attitude control. 


\section{Acknowledgements}

This paper has been sponsored by National 863 Project (2009AA043302). The corresponding author of this paper is Yu Yong, and her email address is yuyong@buaa.edu.cn.

\section{References}

[1] Matthias J.Reiner Johann Bals. Nonlinear inverse models for the control of satellites with flexible structures[C]// International Modelica Conference. 2014:577-588.

[2] Pulecchi T, Casella F, Lovera M, et al. A Modelica Library for Space Flight Dynamics[C]// International Modelica Conference.

[3] Montenbruck O, Eberhard G. Satellite orbits : models, methods, and applications[M]. Applied Mechanics Reviews, 2002.

[4] Duan. Deployment Dynamics of Flexible Solar Arrays Considering Frictional Joint[J]. Chinese Journal of Theoretical and Applied Mechanics. 46(6). 2014. 11: 957-970.

[5] Wang.Dynamic Simulation Analysis of Solar Array Ground Deployment and locking.[J]// Spacecraft Engineering. 20(03). 2011. 5: 86- 92.

[6] Zhao. Computational method of rigid multibody system dynamics considering cylindrical joint clearance[J].Journal of Vibration and shock. 32(17):171-176.

[7] Casella F, Lovera M. High-accuracy simulation of orbital dynamics: An object-oriented approach[J]. Simulation Modelling Practice \& Theory, 2008, 16(8):1040-1054.

[8] ]Xu W, Liu Y, Liang B, et al. Unified multi-domain modelling and simulation of space robot for capturing a moving target[J]. Multibody System Dynamics, 2010, 23(3):293-331.

[9] Xu W. Development of united multi-domain modeling and simulation platfrom for three-axis stabilized spcecrafts.[J].Journal of Harbin institute of technology. , 2011(9):74-80.

[10] Wang H. Research on KeyTechnology of Attitude Control Flywheel System For Small Satellites Application [D] University of Chinese Academy of Sciences. 2015.

[11] Michael Thümmel, Gertjan Looye, Matthias Kurze, et al. Nonlinear Inverse Models for Control[C]// 4th International Modelica Conference. 2005:267-279. 